JORNADAS

\title{
TRATAMIENTO NO FARMACOLÓGICO EN LA ENFERMEDAD RENAL POR DIABETES
}

\author{
NON-PHARMACOLOGICAL TREATMENT \\ IN KIDNEY DISEASE CAUSED BY DIABETES
}

Estrella Menéndez

\begin{abstract}
RESUMEN
El tratamiento no farmacológico de la enfermedad renal crónica (ERC) consiste en regular del consumo de sodio, abandonar el hábito tabáquico, realizar actividad física y lograr un descenso de peso en caso de sobrepeso/obesidad. Deberían tenerse en cuenta el diagnóstico y tratamiento adecuado de la desnutrición y el control de la ingesta de algunos minerales como potasio, fósforo y calcio. Se recomienda un plan de alimentación con proteínas controladas entre 0,8 a $0,9 \mathrm{~g} / \mathrm{kg}$ peso en las personas con enfermedad renal diabética hasta el estadio 5. La evaluación y el monitoreo de la ingesta calórica y de macro y micronutrientes se aconsejan en pacientes con ERC, especialmente en estadios avanzados donde el riesgo de desnutrición es mayor.
\end{abstract}

Palabras clave: enfermedad renal crónica, minerales, proteínas, tabaco.

Revista de la Sociedad Argentina de Diabetes 2017; Vol. 51 (90-93)

\begin{abstract}
The non-pharmacological treatment of chronic kidney disease (CKD) consists in regulating sodium consumption, abandoning cigarette smoking, perform physical activity and attain weight loss in case of overweight/obesity. The diagnosis and adequate treatment of malnutrition and intake control potassium, phosphorus and calcium should be considered. A meal plan with controlled proteins between 0.8 to $0.9 \mathrm{~g} / \mathrm{kg}$ weight is recommended to people with diabetic kidney disease until stage 5. The evaluation and monitoring of dietary consumption and macro and micronutrients recommended in patients with CKD especially in advance stages, where the malnutrition risk is greater.
\end{abstract}

Key words: chronic kidney disease, minerals, proteins, tobacco.
Médica especialista en Nutrición, especializada en Diabetes; Médica del Servicio de Nutrición y Diabetes CEMIC; miembro del Comité de Nefropatía de la Sociedad Argentina de Diabetes, CABA, Argentina

Contacto de la autora: Estrella Menéndez

E-mail: estrellamenendez@yahoo.com
Correspondencia: Conesa $19903^{\circ}$ piso, Depto. C (C1428CUD), CABA, Argentina

Tel: (011) 3641-2271

Fecha de trabajo recibido: 18/08/17

Fecha de trabajo aceptado: 30/08/17

Conflictos de interés: la autora declara que no existe conflicto de interés

\section{Tratamiento no farmacológico}

El tratamiento no farmacológico de la enfermedad renal crónica consiste en:

1) Regulación del consumo de sodio.

2) Abandonar el hábito tabáquico.

3) Realizar actividad física.

4) Descender de peso en estadios tempranos.

5) Diagnóstico y tratamiento de la desnutrición en estadios tardíos.

6) Asesoramiento sobre el consumo de algunos minerales (potasio, fósforo, calcio).

7) Control de la ingesta de proteínas.

\section{Consumo de sodio}

Se recomienda el descenso de la ingesta de sodio a 2 g/día, lo que corresponde a $5 \mathrm{~g}$ de cloruro de sodio o sal común'. En pacientes con enfermedad renal crónica (ERC) la alteración en la excreción de sodio está presente y altas ingestas de sodio incrementan la presión arterial y la proteinuria, inducen hiperfiltración glomerular y alteran la respuesta al bloqueo del sistema renina angiotensina. Por el contrario el descenso del sodio dietario incrementa la efectividad de los $\mathrm{ARA}^{2}$ o de Ios IECA ${ }^{2}$. Una forma habitual de chequear el cumplimiento de esta indicación es la medición del sodio urinario en 24 hs; el hallazgo de $100 \mathrm{Meq}$ de sodio/24 hs se correlaciona con una ingesta de $2.300 \mathrm{mg}$ de sodio/día. 


\section{Hábito tabáquico}

Se ha documentado con estudios poblacionales y metaanálisis la asociación entre fumar y el daño renal en la población general, así como en pacientes hipertensos y/o diabéticos ${ }^{3}$. Este riesgo puede ser hasta cuatro veces mayor a la población no fumadora ${ }^{4}$. Existe fuerte asociación entre el hábito de fumar con eventos cardiovasculares en la población general y el aumento del riesgo de los mismos eventos en pacientes con insuficiencia renal crónica (IRC). Algunos estudios poblacionales demostraron un riesgo aumentado en hombres con respecto a las mujeres ${ }^{4,5}$.

\section{Actividad física}

Se recomienda que pacientes con ERC realicen actividad física compatible con su estado cardiovascular al menos 30 minutos, cinco veces por semana1. En general estas personas presentan una reducción en su capacidad de hacer ejercicio, lo que se asocia con un incremento en la mortalidad y una disminución de la calidad de vida ${ }^{6}$. El ejercicio realizado en forma regular mejora la capacidad aeróbica, reduce la mortalidad cardiovascular, controla de forma más adecuada la diabetes y/o hipertensión y/o dislipemia, mejora las masas ósea y muscular, disminuye la fragilidad de algunos pacientes, mejora la autoestima y la calidad de vida, y desciende los índices de depresión? La indicación debe hacerse desde estadios muy tempranos de la ERC y debiera sostenerse aún durante la diálisis. Se sugiere ejercicio aeróbico y de resistencia ${ }^{8}$.

\section{Descenso de peso}

La obesidad se asocia con un aumento de la morbimortalidad y una disminución de la expectativa de vida ${ }^{9}$. Su presencia aumenta el riesgo de padecer diabetes, hipertensión y dislipemia ${ }^{10}$. Además hay estudios epidemiológicos que demuestran que la obesidad puede ser un factor de riesgo independiente y modificable para ERC11,12. Los estudios de intervención con descenso de peso en pacientes con ERC demostraron una disminu- ción significativa de la tensión arterial, de la proteinuria y albuminuria ${ }^{13,14}$.

La elección de patrones alimenticios como la dieta Dietary Approaches to Stop Hypertension (DASH) o la mediterránea, de probada eficacia en la disminución de la morbimortalidad cardiovascular, son buenos ejemplos de alimentaciones que podrían implementarse en el largo plazo en pacientes con diabetes, riesgo cardiovascular y enfermedad renal para también lograr un descenso de peso. La DASH focaliza el consumo de alimentos bajos en sodio y mantiene un porcentaje reducido de grasas y alto en dulces bajos en sodio (frutas, jugos de frutas, mermeladas), mientras que la mediterránea provee mayor palatabilidad y aceptación con el consumo de aceite de oliva, su alimento paradigmático, así como de frutas secas, aceitunas y palta, a la vez que disminuye mucho el consumo de dulces ${ }^{15}$.

La cirugía bariátrica podría ser una opción en los pacientes que no logran con los cambios de hábitos un descenso de peso sustentable. En un estudio retrospectivo observacional de pacientes con ERC en estadios 3 y 4, operados de by pass gástrico en $Y$ de Roux seguidos durante tres años, se observó una mejoría estadísticamente significativa de la tasa de filtrado glomerular (TFG) en aquellos bariatrizados vs controles, 9,8 $\mathrm{ml} / \mathrm{min}$ mayor que controles (95\% Cl,8,05-11,62). Cada 4,5 kg de descenso de peso la TFG 0,21 ml/min se incrementó en pacientes con manga y $0,44 \mathrm{ml} / \mathrm{min}$ en by pass gástrico ${ }^{16}$.

\section{Evaluación nutricional}

La evaluación y el monitoreo de la ingesta calórica y de macro y micronutrientes a cargo de nutricionistas entrenados en la evolución renal son aconsejables en pacientes con ERC especialmente en estadios avanzados, donde el riesgo de desnutrición es más marcado ya que se estima que hasta el $75 \%$ de los pacientes en diálisis está malnutrido. La etiología de la malnutrición en la nefropatía es compleja y multifactorial e intervienen factores nutricionales, metabólicos, hormonales, 
inflamatorios y socioeconómicos. La disminución de la ingesta es posiblemente la causa más importante, dada la "anorexia urémica", la acidosis metabólica, la diálisis per se, las enfermedades intercurrentes y la depresión ${ }^{17}$. Debe considerarse un VCT de $35 \mathrm{cal} / \mathrm{kg}$ de peso en adultos y $30 \mathrm{cal} / \mathrm{kg}$ de peso en mayores de 70 años ${ }^{18}$.

\section{Asesoramiento sobre la ingesta de potasio/fósforo/calcio}

Cuando la función renal declina el riñón pierde la habilidad de eliminar el exceso de potasio, fósforo y calcio. Si el paciente en estadios avanzados presenta hiperkalemia (potasio $>5,5$ ) debe ser asesorado sobre los alimentos ricos en este mineral y las técnicas culinarias que disminuyan el mismo en los alimentos fuente. Tanto un exceso como un déficit de potasio pueden provocar efectos adversos como arritmias o muerte súbita, por lo cual la restricción de este mineral no debe implementarse si no existe evidencia de hiperkalemia ${ }^{19}$. El fósforo es un mineral a tener en cuenta dado que predispone a alteraciones del metabolismo óseo, incluyendo hiperparatiroidismo secundario. El control del fósforo es importante si los niveles de PTH son $>70 \mathrm{pg} / \mathrm{ml}$ en estadio 3 y de $>110 \mathrm{pg} / \mathrm{ml}$ en estadio 4, en dos o más determinaciones. Se recomienda una ingesta de 800 a $1.000 \mathrm{mg}$ de fósforo por día o 10/12 mg de fósforo por gramo de proteínas. En estadios 3 a 5 la ingesta de calcio elemental no debe exceder los 2.000 mg/día considerando el provisto por las comidas y los suplementos que el paciente pueda consumir20.

\section{Ingesta de proteínas}

A diferencia de la ingesta de hidratos y grasas que no impactan sobre la función renal, la proteína de origen animal (especialmente carne roja y subproductos) modula la hemodinámica renal al aumentar el flujo plasmático renal y elevar la presión intraglomerular lo que produce hiperfiltración renal en agudo y conduce al acúmulo de metabolitos tóxicos en forma crónica. A pesar que el descenso en la cantidad de proteínas ingeridas es muy importante en los pacientes con enfermedad renal no diabética, esto no ha podido demostrarse en pacientes con nefropatía diabética. En los metaanálisis de Pan et al. ${ }^{21}$ y Robertson et al. ${ }^{22}$ se incluyeron personas con diabetes que presentaban proteinuria o albuminuria, con planes de alimentación bajos en proteínas (media 0,9 $\mathrm{g} / \mathrm{kg}$ peso) vs ingesta proteica habitual (media $1,27 \mathrm{~g} / \mathrm{kg}$ peso), y no se encontró diferencia significativa entre ambos grupos en el descenso de la TFG. Pan tampoco halló diferencias en la excreción de proteínas, pero un tema de preocupación aportado por este metaanálisis fue el estado nutricional con las dietas restrictas en proteínas, ya que se demostró que las ingestas más bajas en proteínas provocaban descensos significativos de la albumina sérica. Este descenso no se observa con ingestas superiores a 0,7 g/kg peso por lo que se recomienda un plan de alimentación con proteínas controladas entre 0,8 a 0,9 g/kg peso en las personas con enfermedad renal diabética hasta el estadio 5 (TFG <15). En 2015 la Academia de Nutrición y Dietética concluyó que la cantidad de proteínas ingeridas en un rango entre 0,7 a $2 \mathrm{~g} / \mathrm{kg}$ peso no tiene efecto sobre la TFG independiente del descenso de peso en adultos con diabetes tipo 1 ó tipo 2 con $\mathrm{ERC}^{23}$. Con respecto al efecto de las proteínas vegetales sobre la función renal, en el estudio de Azadbakht et al. con 41 pacientes con DM2 y macroalbuminuria seguidos durante cuatro años divididos en dos grupos, en uno de ellos con $70 \%$ de proteínas animales y el otro con un $30 \%$ de proteína de soja, el grupo con proteína vegetal tuvo menores niveles de proteína $\mathrm{C}$ reactiva, de colesterol total, LDL y triglicéridos. También se observó un descenso en la proteinuria y creatininuria sin variación en la TFG entre ambos grupos ${ }^{24}$. En pacientes con una TFG $<20 \mathrm{ml} / \mathrm{min}$ se sugiere indicar un descenso en la ingesta proteica de $0,8 \mathrm{~g} / \mathrm{kg} / \mathrm{dia}^{25}$. No es claro el efecto que las restricciones proteicas más importantes tienen en la progresión de la ERC, pero en estadio 
5 ó predialítico su implementación con el uso de alimentos vegetales, algunos de ellos modificados como el arroz aproteico o las pastas y panes hechos sin proteínas, pueden generar un estado de bienestar por la disminución de los tóxicos producidos por la proteína animal26. El descenso a 0,6g/kg/día debería hacerse con un estricto seguimiento nutricional y posiblemente con el uso de cetoanálogos. Su empleo en pacientes muy añosos con $\mathrm{TFG}<10 \mathrm{ml}$ demostró ser efectiva en prolongar la llegada al tratamiento sustitutivo ${ }^{27}$.

\section{BIBLIOGRAFÍA}

1. KDIGO 2012. Clinical practice guideline for the evaluation and management of chronic kidney disease. Kidney Int Supl 2013;3:1.

2. Jones-Burton C, Mishra SI, Fink JC, et al. An in depth review of evidence linking dietary salt intake and progression of chronic kidney disease. Am J Nephrol 2006; 26(3):268-75.

3. Orth SR, Hallan SI. Smoking: a risk factor for progression of chronic kidney disease and for cardiovascular morbidity and mortality in renal patients absence of evidence or evidence of absence? Clin J Am Soc Nephrol 2008; 3(1):226-36.

4. Hallan SI, Orth SR. Smoking is a risk factor in the progression to kidney failure. Kidney Int 2011; 80(5):516523.

5. Sawicki PT, Didjurgeit U, Muhlhauser I, et al. Smoking is associated with progression of diabetic nephropathy. Diabetes Care 1994; 17(2):126-31.

6. Beddhu S, Baird B, Zitterkoph, et al. Physical activity and mortality in chronic kidney disease (NHANES III) Clin J Am Soc Nephrol 2009; 4:1901-1906.

7. Padilla J, Krasnoff J, Da Silva M, et al. Physical functioning in patients with chronic kidney disease. J Nephrol 2008; 21:550-559.

8. Johansen K, Chertow G, Ng A, et al. Physical activity levels in patients on hemodialysis and healthy sedentary controls. Kidney Int 2000; 57(6):2564-70.

9. Hall JE, Crook ED, Jones DW, et al. Mechanisms of obesity associated cardiovascular and renal disease. Am J Med Sci 2002; 324(3):127-37.

10. Wahba IM, Mak RH. Obesity and obesity initiated metabolic síndrome: mechanistic links to chronic kidney disease. Clin J Am Soc Nephrol 2007; 2(3):550-62.

11. Hobbs H, Farmer $\mathrm{C}$, Irving J, et al. Is high body mass index independently associated with diminished glomerlar filtration rate? An epidemiological study. J Renal Care 2011; 37(3):148-154.
12. Wang Y, Chen X, Song Y, et al. Association between obesity and kidney disease: a sistematic review and meta analysis. Kidney Int 2008; 73(1):19-33.

13. Navaneethan SD, Yehnert H, Moustarah F, et al. Weight loss interventions in chronic kidney disease: a sistematic review and meta-analysis. Clin J Am Soc Nephrol 2009; 4(9):1565-74.

14. Afshinnia F, Wilt RJ, Duval S, et al. Weight loss and proteinuria: systematic review of clinical trials and comparative cohorts. Nephrol DialTransplant 2010; 25(4):1173-83.

15. Gallieni M, Cupisti A. DASH and Mediterranean Diets as nutritional interventions for CKD patients. Am J Kidney Dis 2016; 68(6):828-830.

16. Imam TH, Fischer H, Jing B, et al. Estimated GFR before and after bariatric surgery in CKD. Am J Kidney Dis 2017; 69(3):380-388.

17. Ollero D, Riobo P, Sánchez Vilar O, et al. Soporte nutricional en la insuficiencia renal crónica. Endocrinol Nutr 2005; 52(supl 2): 5-58.

18. KDOQI 2007. Clinical practice guidelines and clinical practice recommendations for diabetes and CKD disease. Guideline 5: nutritional management in diabetes and chronic kidney disease. Am J Kidney Dis 2007; 49:2 (suppl 2) S95-S107.

19. Riella M, Martins C. Nutrición en la progresión de la insuficiencia renal crónica. En: Nutrición y riñón, $2^{\circ}$ Ed. Editorial Panamericana 2006; cap. 10, 97-109.

20. Academy of Nutrition and Dietetics Evidence Analysis Library. Chronic kidney disease evidence based nutrition practice guidelines. 2010. Disponible en: http://www.andel.org.

21. Pan Y, Guo LL, Jin HM. Low protein diet for diabetic nephropathy: a meta-analysis of randomized controlled trials. Am J Clin Nutr 2008; 88(3):660-6.

22. Robertson L, WaughN, Robertson A. Protein restriction for diabetic renal disease. Cochrane Database Syst Rev 2007; 17(4)CD002181.

23. Academy of Nutrition and Dietetics Evidence Analysis Library. Diabetes type 1 and 2 evidence based Nutrition practice guidelines for adults. 2015. Disponible en: http://www.andel.org.

24. Azadbakht L, Atabak S, Esmailizadeh A. Soy protein, cardiorenal índices and creactive protein in type 2 diabetes with nephroparhy. Diabetes Care 2008; 31:648-654.

25. Piccoli GB, Ventrella F, Capizzi I, et al. Low protein diets in diabetic chronic kidney disease (CDK) patients: are they feasible and worth the effort? Nutrients 2016; 8(10):649

26. Piccoli GB, Vigotti FN, Leone F, et al. Low protein diets in CKD: how can achieve them? A narrative, pragmatic review. Clin Kidney J 2015; 8(1):61-70.

27. Kiuchi A, Ohashi Y, Tai R, et al. Association betwen low dietary protein intake and geriatric nutrition risk index in patients with chronic kidney disease. A retrospective single center cohort study. Nutrients 2016; 8(10):662. 